\title{
The interplay between radio-activity and the ISM in radio galaxies
}

\author{
R. Morganti ${ }^{1}$ \\ ${ }^{1}$ Netherlands Foundation for Research in Astronomy, Postbus 2, NL-7990 AA, Dwingeloo, NL \\ email: morganti@astron.nl
}

\begin{abstract}
Radio-loud AGNs can inhabit regions with a very rich ISM. The presence of this rich medium is likely related to the origin and evolution of the host galaxy and of the active nucleus. Recent observations show that a large fraction of radio galaxies contains a significant young stellar population. This supports the idea that mergers are responsible for both the starburst phase and the triggering of the nuclear activity. The gas that reaches the central regions can have quite disturbed kinematics, likely due to the effects of the AGN activity and in particular of the powerful radio jets. The recent detection of fast nuclear gas outflows, observed both in ionised and neutral gas, is giving new and important insights into the physical conditions of the gaseous medium around the nucleus and the interaction between the AGN and this medium. Finally, as another example of the interplay between the radio activity and the ISM, the possibility of star formation induced by the passage of the radio jet will be discussed.
\end{abstract}

\section{Introduction}

This review concentrates on one particular type of active nucleus, radio galaxies, where the presence of large-scale and powerful jets makes it particularly interesting to look for the importance of the interplay between these structures and the interstellar medium (ISM). Radio galaxies are invariably associated with early-type host galaxies, i.e. galaxies that were thought until not so long ago to have a relatively uninteresting ISM. It is clear now that at least a fraction of them has a very rich ISM likely related to their origin.

The origin of activity in galaxies is often explained as triggered by merger and/or interaction processes. Torques and shocks during the merger can remove angular momentum from the gas in the merging galaxies and this provides injection of substantial amounts of gas/dust into the central nuclear regions (see e.g. Mihos \& Hernquist 1996). This also appears to be the case for radio galaxies as suggested by morphological and kinematical evidence (e.g. Heckman et al. 1986, Tadhunter et al. 1989). On the other hand, the phase of nuclear activity triggered in this way is now increasingly recognised to play an important role in the evolution of the galaxy itself. Particularly important in this respect are gas outflows that can be generated by this activity and the effect they can have on the interstellar medium (ISM). This feedback can be extremely important for the evolution of the galaxy, up to the point that it could limit the growth of the nuclear black-hole (e.g. Silk \& Rees 1998, Wyithe \& Loeb 2003). Thus, the processes of assembly of the host galaxy, the supply of gas to the central region, as well as the effects that the triggering of the (radio) activity has on this gas, are tightly related and essential for our understanding of radio galaxies.

Here I will review some recent results related to these topics and in particular I will discuss two possible effects of the interplay between radio activity and ISM: gaseous outflows and jet induced star formation. 


\section{Starburst, AGN phase and the order of events}

The origin of radio galaxies can be studied using the stellar population of the host galaxy. This gives information on the age of the last merger, as well as the type of merger that triggered the activity. Complementary to this is the study of the large-scale $\mathrm{HI}$, that can be used as another tracer of the formation process of their host galaxy.

\subsection{Results from the stellar population}

A number of surveys have shown - through the modelling of spectroscopic and polarimetric data - that young stellar populations (YSP) make a significant contribution to the optical/UV continua in $25-40 \%$ of powerful radio galaxies at low and intermediate redshift $(z<0.7$, Aretxaga et al. 2002, Tadhunter et al. 2002, Wills et al. 2002, 2004). Similar results have been obtained also from $U V$ imaging (Allen et al. 2002).

Furthermore, the study of a few selected radio galaxies (3C 293, 3C 305 and 4C12.50, Tadhunter et al. 2004) has shown that the YSP are relatively old (0.1 - 2 Gyr), massive $\left(10^{9}<M_{\mathrm{YSP}}<5 \times 10^{10} M_{\odot}\right)$ and make up a large proportion $(\sim 1-50 \%)$ of the stellar content. These results are consistent with the idea that AGN activity, at least in some radio galaxies, is triggered by major gas-rich mergers, and confirm that an evolutionary link exists between radio galaxies and luminous- and ultra-luminous infrared galaxies (Tadhunter et al. 2004) as suggested earlier e.g., by the study of the molecular gas (see e.g. Evans et al. 1999). These mergers will trigger both the starburst and the AGN activity. Interestingly, these results also suggest that the radio activity is triggered relatively late in the merger sequence. This delay could be due to the fact that the starburst phase momentarily clears the central regions from gas, and that it takes some time before other "fuel" can concentrate again in the nuclear regions and trigger, this time, the AGN. However, the fact that a large fraction of radio galaxies do not show a young stellar population component also indicates (as previously suggested e.g. Heckman et al. 1986) that radio sources are not triggered by a single type of merger or at a particular stage of the merger sequence (Wills et al. 2002).

\subsection{Results from $H$ I studies}

A further way to investigate the origin and evolution of the host galaxy is by studying the large scale neutral hydrogen. As already found in "normal" early-type galaxies (see e.g. Oosterloo et al. 2004), some radio galaxies also show very large (up to $200 \mathrm{kpc}$ in size and masses up to $10^{10} M_{\odot}$ ) and regular disks (of low column density) neutral hydrogen (see e.g. the case of PKS B1718-649, Véron-Cetty et al. 1995 and B2 0648+27, Morganti et al. 2003a). A systematic survey (Emonts et al. in prep.) is now looking for more cases like these. One example, the large H I disk detected around the compact radio galaxy B2 $0258+35$, is shown in Fig. 1. Given their size and regular appearance, these disks must be quite old ( $>10^{9} \mathrm{yr}$ ), hence they are not related to recent accretions, in agreement with what is found from the study of the stellar population. Thus, also from the study of the HI we derive that the radio activity starts late after the merger. Interestingly, all these large and rich H I disks appear to be, so far, associated with compact (steep spectrum) radio sources, typically considered young radio galaxies (O'Dea 1998).

\section{Outflows of neutral and ionized gas in the central regions}

Given the origin described above of, at least some, radio galaxies, one can expect large amounts of gas to be transported and concentrated in the central regions. Examples of high nuclear concentration of gas, in particular molecular gas, are indeed known (e.g. Evans et al. 1999, 2004). The ionised and neutral gas observed in the central regions 


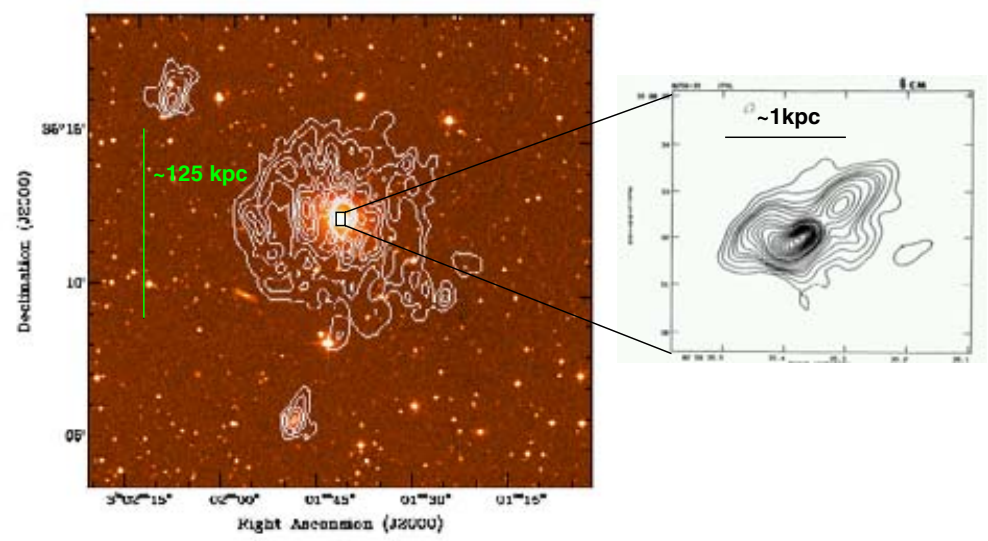

Figure 1. Left: H I total intensity (contours) superimposed on an optical image of B2 0258+35 (from Emonts et al. in prep.). Right: The continuum image (from Fanti et al. 1986).

of radio galaxies is often associated with circum-nuclear disks, as already discussed in other contributions in these Proceedings. However, gas is not only observed in these "settled" structures. In young (i.e. CSS and GPS, O'Dea 1998) or recently re-started radio galaxies, a cocoon of gas may still surround the active nucleus and the effects produced on it by the AGN activity can be observed. For example, the radio jets are expected to go through some "struggle" to be able to emerge from the nuclear regions of these young radio sources. Indeed, broad optical (forbidden) emission lines are typically observed in CSS/GPS sources, indicating the presence of gas with disturbed kinematics as a result of an interaction of the radio plasma with the ISM (Gelderman \& Whittle 1994, O’Dea et al. 2002).

In addition to this, gaseous outflows are now unambiguously detected in a number of young radio sources. The two best examples are PKS 1549-79 and 4C 12.50 (Tadhunter et al. 2001, Holt et al. 2003). In the case of 4C 12.50, complex, multi-component emission line profiles are observed at the position of the nucleus. The broadest component has FWHM $2000 \mathrm{~km} \mathrm{~s}^{-1}$, is blueshifted by $\sim 2000 \mathrm{~km} \mathrm{~s}^{-1}$ with respect to the halo of the galaxy, has a large reddening and high density $\left(n_{\mathrm{e}}>4200 \mathrm{~cm}^{-3}\right)$. This component is therefore interpreted as the material closer to the radio jet and interacting with it. This interaction produces the observed outflow. A more quiescent (and extended) component of ionized gas is also observed and it is associated with the cocoon of gas surrounding the radio source, but not yet interacting with it. Thus, 4C 12.50, as well as PKS 1549-79, appears to be a young radio galaxy with nuclear regions that are still enshrouded in a dense cocoon of gas and dust. The radio jets are now expanding through this cocoon, sweeping material out of the nuclear regions (Holt et al. 2003). Interestingly, high resolution (VLBI) observations of the $\mathrm{HI}$ in $4 \mathrm{C} 12.50$ show that deep and relatively narrow $\mathrm{H}$ I absorption (observed at the systemic velocity) is indeed associated with an off-nuclear cloud $(\sim 50$ to $100 \mathrm{pc}$ from the radio core) with a column density of $\sim 10^{22} T_{\text {spin }} /(100 \mathrm{~K}) \mathrm{cm}^{-2}$ and an $\mathrm{HI}$ mass of a few times $10^{5}$ to $10^{6} M_{\odot}$. Although this gas will not be able to confine the radio source, it could, however, be able to momentarily destroy the path of the jet, as shown also by numerical simulations (Bicknell et al. 2003). Thus, this interaction can influence the growth of the radio source until the radio plasma clears its way out. It is interesting to point out the fact that all cases of fast outflows (both of ionised and neutral gas, see below) have been observed so far in radio galaxies with YSP. Thus, this could indicate that the galaxy is indeed in a particular stage of its evolution, where 

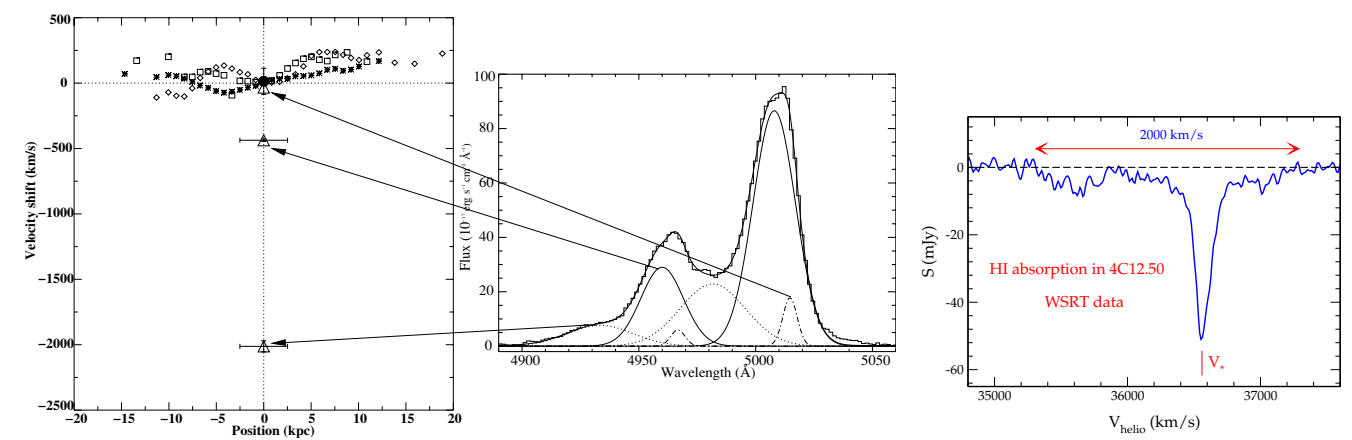

Figure 2. Left: Radial velocity profile of the extended gaseous halo of $4 \mathrm{C} 12.50$ (from Holt et al. 2003). Key: $*, \diamond$ and $\square$ : highly extended [O II] emission (narrowest component); •: H I 21-cm absorption; $\triangle: 3$ components of [O III] in the nucleus. The [OIII] profile with the components from the fit is also shown. Right: The HI profile (from the WSRT) of 4C 12.50 showing the broad absorption. The spectra are plotted in flux density (mJy) against the optical heliocentric velocity in $\mathrm{km} \mathrm{s}^{-1}$.

large amounts of gas are still present in the central region. The hollowed out bi-conical structures observed, e.g., in the nearby radio galaxy Cygnus A (Tadhunter et al. 1999), might represent the aftermath of this process once the source reaches a later stage of the evolution.

\subsection{Fast outflows of neutral hydrogen}

Extremely intriguing is the discovery of a number of radio galaxies where the presence of fast outflows also is associated with neutral gas. The best example of this phenomenon found so far is the radio galaxy 3C 293 (Morganti et al. 2003b), but more cases have now been observed (see also Oosterloo et al. these Proceedings). Fig. 2 shows the case of $4 \mathrm{C}$ 12.50. The broad and shallow (with optical depth typically only a fraction of a \%) HI absorption features detected against the central regions have a full width at zero intensity ranging between 800 and $2000 \mathrm{~km} \mathrm{~s}^{-1}$, mostly blueshifted relative to the systemic velocity. The broad H I absorption represents a fast outflow of neutral gas from the central regions of these radio galaxies. Interestingly, in the few cases known so far, the fast outflows detected in neutral and ionised gas show similar kinematics (Oosterloo et al. these Proceedings). This suggests that both are due to the same mechanism.

Gas outflows of ionised gas are detected in other types of AGN (such as quasars and Seyfert galaxies, see e.g. Kriss these proceeding). These outflows can be produced in regions close to the accretion-disk of the AGN (Elvis 2000), or the gas could be accelerated through radiation and/or wind pressure originating in dusty narrow-line regions (Grove et al. these Proceedings) or starburst winds (Heckman et al. 1990). However, for radioloud objects, gas outflows can be due to the interaction between the radio plasma and the ISM medium. To discriminate between all these mechanisms as the origin for the outflows in radio galaxies, it is necessary to identify the location where the outflow (both in ionised and neutral gas) is occurring in relation to the radio structure. So far this has been done only in the case of 3C 293 (see Oosterloo et al. these Proceedings) where the outflow appears to be located at the position of a radio lobe. A possible model of what is happening is that the radio plasma jet hits a (molecular) cloud in the ISM. As a consequence of this interaction, part of the gas is ionised and its kinematics is disturbed by the shock. Once the shock has passed, part of the gas may have the chance to recombine and become neutral, while it is moving at high velocities. A similar explanation has been proposed for other objects (see e.g. Conway \& Schilizzi 2002). As a result of such an 


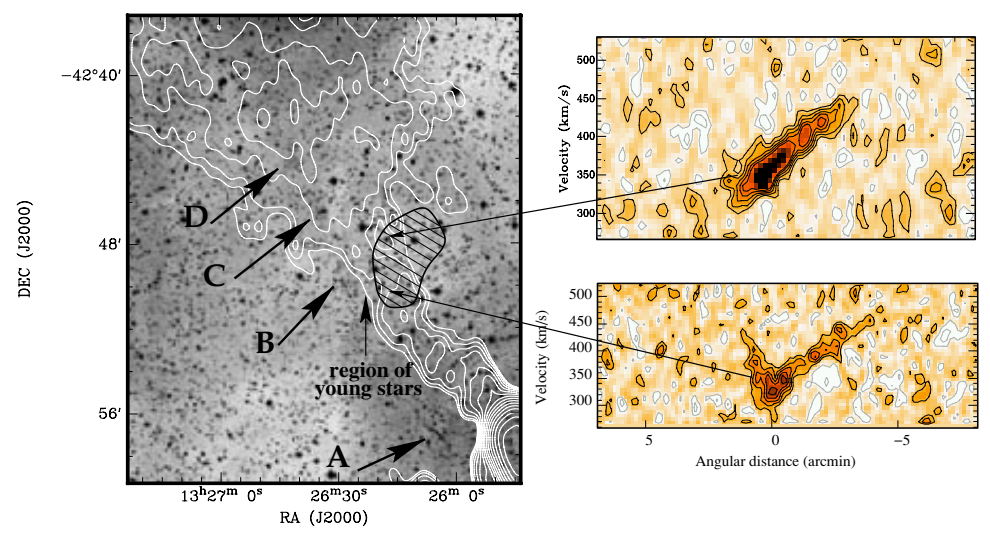

Figure 3. Left: The radio continuum (contours) superimposed to an optical image of Centaurus A (from Morganti et al. 1999, the regions of the optical filaments are labelled). The region with H I emission is shaded. Right: two position-velocity diagrams showing (top) the smooth, regular velocity pattern observed in most of the cloud and (bottom) the region where anomalous velocities are observed.

interaction, star formation might also occur (see below). Finally, it is worth to point out that these cases of extreme kinematics of the gas indicate once again that (as pointed out in more detail in other talks at this conference) one has to be very careful in using either the neutral or the ionised gas to derive black hole masses.

\section{Jet-induced star formation}

Another effect of the interplay between the AGN activity and the ISM is star formation induced by the passage of a radio jet. This is particularly relevant, as it has been considered as a possible mechanism to explain the UV continuum emission observed in the host galaxies of high- $z$ radio galaxies and the "alignment effect" between radio emission and this continuum (Rees 1989).

Recent numerical simulations have shown that the interaction of a radio jet with a clumpy medium can produce fragmented clouds that can quickly cool and condense (see Mellema et al. 2002, Fragile et al. 2004). Thus, the interaction and the consequent production of shocks from the radio jet (even with moderate velocity) has, indeed, the potential to trigger large-scale star formation in a galaxy, (Fragile et al. 2004). Few cases are known in nearby radio galaxies (see e.g. van Breugel et al. 2004) and one of the best example is observed in the radio galaxy Centaurus A. The detection of groups of young stars in the northern lobe of this galaxy and close to the location of the radio jet, has been explained by different authors as due to gas shocked by the passage of this jet (see e.g. Graham 1998). Next to this region ( $15 \mathrm{kpc}$ from the centre) an H I cloud has also been observed (Schiminovich et al. 1994). This cloud has been interpreted as being part of a single inclined ring regularly rotating in a circular orbit. However, observations of this cloud with higher velocity resolution (Oosterloo \& Morganti 2004 in prep.) reveal anomalous velocities of the HI. The position-velocity diagram shown in Fig. 3 shows that an abrupt change in velocity, of about $100 \mathrm{~km} \mathrm{~s}^{-1} \mathrm{kpc}^{-1}$, happens in the southern tip of the cloud, i.e. the region closer (in projection) to the passage of the radio jet. Such change in velocity can only be explained as the result of the interaction between the jet and the HI cloud. This is interpreted as further evidence that, at that location, an interaction is occurring between the radio jet and the ISM. This gives further evidence 
that the young stars observed in that region are the result of such an interaction, and that the neutral hydrogen provides the reservoir that makes the star formation possible.

\section{Acknowledgements}

I would like to thank the organisers, in particular Thaisa Storchi Bergmann, for inviting me and for the financial support. I am also very grateful to my collaborators, in particular Tom Oosterloo and Clive Tadhunter, with whom I have done a significant fraction of the work discussed here.

\section{References}

Allen, M., Sparks, W. B., Koekemoer, A., \& Martel, A. R. 2002, ApJS, 139, 411

Aretxaga, I., et al. 2002, MNRAS, 325, 636

Bicknell, G., Saxton, C. J., Sutherland, R. S., Midgley, S., \& Wagner, S. J. 2003, New Astronomy Reviews, 47, 537

Conway, J. E., \& Schilizzi, R. T. 2000, in 5th European VLBI Network Symposium, (eds. J.E. Conway et al.) Onsala Space Observatory, p. 123

Elvis, M., Marengo, M., \& Karovska, M. 2002, ApJ, 576, L106

Evans, A., et al. 2004, in "Neutral ISM in Starburst Galaxies", (eds. S. Aalto, S. Huttemeister \& A. Pedlar), ASP, in press

Evans A., Kim J. M., Mazzarella J. M., Scoville N. Z., \& Sanders D. B. 1999, ApJ, 521, L107

Fanti, C., et al. 1986, A\&AS, 65, 145

Fragile, P. C., Murray, S., Anninos, P., \& van Breugel, W. 2004, ApJ, 604, 74

Gelderman, R., \& Whittle, M. 1994, ApJS, 91, 491

Graham, J. A. 1998, ApJ, 502, 245

Heckman, T. M., et al. 1986, ApJ, 311, 526

Heckman, T. M., Armus, L., \& Miley, G.,K. 1990, ApJS, 74, 833

Holt, J., Tadhunter, C., \& Morganti R. 2003, MNRAS, 342, 227

Mellema, G., Kurk, J. D., \& Röttgering, H. 2002, A\&A, 395, L1

Mihos, J. C., \& Hernquist, L. 1996 ApJ, 464, 641

Morganti, R., Killeen, N. E. B., Ekers R. D., \& Oosterloo, T. A. 1999, MNRAS, 307, 750

Morganti, R., et al. 2003a, A\&A, 399, 511

Morganti, R., et al. 2003b, ApJ, 593, L69

Morganti R., et al. 2004, A\&A, submitted

O'Dea, C. P. 1998, PASP, 110, 493

O'Dea, C. P., et al. 2002, AJ, 123, 2333

Oosterloo, T. A., et al. 2004, in Recycling Intergalactic and Interstellar Matter, IAU 217, (eds. P.-A. Duc, J. Braine, and E. Brinks), in press (astro-ph/0310632)

Rees, M. J. 1989, MNRAS, 239, 1P

Schilizzi, R., et al. 2001, A\&A, 368, 398

Schiminovich, D., van Gorkom, J. H., van der Hulst, J. M., \& Kasow, S. 1994, ApJ, 423, L101

Silk, J., \& Rees, M. J. 1998, A\&A, 331, L1

Tadhunter, C., Fosbury, R. A. E., \& Quinn, P. 1989, MNRAS, 240, 225

Tadhunter, C., et al. 1999, ApJ, 512, L91

Tadhunter, C. N., Wills, K. A., Morganti, R., Oosterloo, T., \& Dickson, R. 2001, MNRAS, 327, 227

Tadhunter, C. N., et al. 2002, MNRAS, 330, 977

Tadhunter, C. N., et al. 2004, MNRAS, submitted

van Breugel, W., et al. 2004, in Recycling Intergalactic and Interstellar Matter, IAU 217 (eds. P.-A. Duc, J. Braine, and E. Brinks), in press (astro-ph/0312282)

Véron-Cetty, M.-P., Woltjer, L., Ekers, R. D., \& Staveley-Smith, L. 1995, A\&A, 297, L79

Wills, K. A., Tadhunter, C. N., Robinson, T. G., \& Morganti, R. 2002, MNRAS, 333, 211

Wills, K. A., et al. 2004, MNRAS, 347, 771

Wyithe, J. S., \& Loeb, A. 2003, ApJ, 595, 614 